\title{
A TRANSMISSION/ESCAPE PROBABILITIES \\ MODEL FOR NEUTRAL PARTICLE TRANSPORT IN THE OUTER REGIONS OF A DIVERTED TOKAMAK
}

\author{
W. M. Stacey
}

Fusion Research Center and

Nuclear Engineering Program

Georgia Institute of Technology

Atlanta, GA 30332 USA

December, 1992

\section{DISCLAIMER}

\begin{abstract}
This report was prepared as an account of work sponsored by an agency of the United States Government. Neither the United States Government nor any agency thereof, nor any of their employees, makes any warranty, express or implied, or assumes any legal liability or responsibility for the accuracy, completeness, or usefulness of any information, apparatus, product, or process disclosed, or represents that its use would not infringe privately owned rights. Reference herein to any specific commeritial product, process, or service by trade name, trademark, manufacturer, or otherwise does not necessarily constitute or imply its endorsement, recommendation, or favoring by the United States Government or any agency thereof. The views and opinions of authors expressed herein do not necessarily state or reflect those of the United States Government or any agency thereof.
\end{abstract}




\begin{abstract}
A new computational model for neutral particle transport in the outer regions of a diverted tokamak plasma chamber is presented. The model is based on the calculation of transmission and escape probabilities using first-flight integral transport theory and the balancing of fluxes across the surfaces bounding the various regions. The geometrical complexity of the problem is included in precomputed probabilities which depend only on the mean free path of the region.
\end{abstract}




\section{INTRODUCTION}

The transport of neutral atoms and molecules of fuel and impurity species in the "outer regions"--the edge plasma, the scrape-off layer (SOL), the divertor channels, and the plasma chamber and divertor plenums--of a diverted tokamak is a computational problem the practical significance of which is growing in recognition. There are indications that conditions in the plasma edge may control the energy confinement of the bulk plasma, and such conditions in turn may be influenced by the neutral population in the outer regions. More directly, the interaction among neutrals and plasma ions and electrons in the outer regions is being relied on to "radiatively cool" the latter and thereby reduce the heat load on the divertor collection plate, which is presently predicted to be excessive in next-generation devices such as ITER.

The importance of neutral particle transport in the outer regions of plasmas has long been recognized by specialists, and a variety of computational models have been used, as reviewed in Ref. [1]. 'Thus, it is incumbent upon anyone who would introduce a new computational model, which is the purpose of this paper, to comment upon the existing models and to indicate why a new model is needed.

There are three general categories of neutral transport computational models, based on: 1) approximation to the integral form of the transport equation; 2) approximation to the differential form of the transport equation; or 3) simulation of particle transport by following a large number of randomly-generated particle histories and statistically averaging the results (Monte Carlo).

The integral transport equation [2] states that, for a given source of particles, those particles going in a given direction are exponentially attenuated as the integral of the inverse mean-free-path ( $\mathrm{mfp}$ ) along the direction of flight. For a fixed source, solution is a straightforward matter of geometry. However, when one of the processes that attenuates the neutrals results in other neutrals with different energy and direction, this process constitutes a secondary neutral source which is distributed in space, and 
so on for tertiary, quartiary, etc. neutral sources. Charge-exchange is one such process for neutrals in the outer regions of a plasma. Thus, the solution of the integral transport equation must proceed iteratively, with an approximate evaluation of the distributed source and an exact solution of the particle transport from the approximate source constituting the iteration step. Because of the coupling among all spatial points that is inherent to the integral transport formulation, this iterative solution can become very computationally intensive for any but the simplest situations. In practice (e.g. [3], the integral transport solution is obtained for the attenuation of particles coming from a fixed source, such as reflection from the wall, and secondary distributed sources are neglected. Such a treatment is satisfactory when the ionization rate is much greater than the charge-exchange rate, but is poor when the two rates are comparable, which is the case in many parts of the outer regions of a plasma.

Approximations based on the differential formulation of the transport equation have been highly developed for neutron transport in connection with nuclear reactor calculations, where they proved to be more practically useful than approximations based on the integral formulation, in general. Two general classes of approximation were developed, distinguished by the treatment of the angular dependence. In the spherical harmonics, or $P_{n}$, method (e.g. [4]), the angular dependence of the neutral distribution is expanded in spherical harmonics, and moments equations are generated by integrating over angle and making use of orthogonality relations. In principle, any degree of angular anisotropy an be treated by taking enough moments. In practice, these methods found their greatest application in one-dimensional problems where a few Legendre polynomials sufficed to represent the angular distribution. In the discrete ordinates, or $S_{n}$, method (e.g. [5]), the transport equation is only solved at a few angular directions (ordinates), with integrals over angle that enter these equations being approximated by a quadrature involving values at these ordinates and a quadrature weight, the choice of which is quite important. When the angular 
distribution can be represented by a linearly anisotropic spherical harmonics approximation or by a two-ordinate discrete ordinates approximation, the lowest order approximation that results is diffusion theory (e.g. [6]. These approximations to the differential transport equation also must be solved iteratively, but because the coupling among spatial points is nearest-neighbor, the iterative procedure is more tractable than for the integral formulation. The diffusion approximation is widely used in neutron transport for 1D, 2D and 3D calculations in which the linear anisotropy assumption is valid, and higher order discrete ordinates approximations are the method commonly employed in 1D and 2D problems when higher order anisotropy in the angular distribution must be included.

Discrete ordinates codes that had been developed for neutron transport were applied to calculate neutral transport in the edge of simple plasma models represented by slab or cylindrical geometry several years ago (e.g. [7]-[9]), but this method does not seem to have been applied recently or extended to the more complex geometry of the outer regions of a diverted tokamak plasma. The diffusion approximation has been applied to plasma outer region calculations recently (e.g. [10]), but the diffusion theory assumption of linear anisotropy in the angular distribution would seem to be invalid in many cases of interest (e.g. the reflection of ions from a wall as neutrals, the interface between a divertor channel in which there is ionization and charge-exchange and a plenum region in which there is free-streaming). We are not aware of an application of $\mathrm{P}_{\mathrm{n}}$ or double- $\mathrm{P}_{\mathrm{n}}$ methods. Double $\mathrm{P}_{1}$ rnethods, in which the angular distribution is assumed isotropic within each of two hemispheres, would seem to offer significant advances in accuracy over diffusion theory in 1D situations. Because of the inherent 2D geometry and angular anisotropy, the discrete ordinates methods would seem to be the best suited of the approximations based on the differential transport formulation for application to neutral transport in the plasma outer regions. 
Monte Carlo calculations simulate the fates of many, randomly generated particles to obtain an averaged solution for the transport of a population of neutral particles. This methodology (e.g. [11]) is highly developed, and, because of previous emphasis upon applications to complex geometries, has good capability to represent the geometrical complexity of the plasma and divertor chambers. Monte Carlo has been extensively applied to the computation of neutral particle transport in the outer regions of diverted tokamaks (e.g. $[12,13])$. Extreme high accuracy can be obtained with Monte Carlo, provided the basic reacticn rate parameters are well known and provided that a sufficient number of histories are simulated that the statistical uncertainty in the averaged solution is acceptably small. This latter proviso can lead to particulariy demanding computational requirements when the Monte Carlo solution is part of a neutral-charged particle iterative solution procedure, since convergence of the outer neutral-charged particle iteration can be destroyed by statistical fluctuations in the Monte Carlo solutions on successive iterates. Monte Carlo will probably remain as the ultimate method for neutral particle transport in diverted tokamak plasmas in cases where high accuracy in a complex geometry is required and computational time is a secondary consideration. However, for routine calculations, the discrete ordinates method and the method to be presented in this paper would seem to have certain computational advantages.

The method which is proposed in this paper utilizes the integral transport method to do what is does best, calculate the uncollided neutral flux that is transmitted from one surface to another. The secondary source in the intervening region is then found by subtraction, and the collided neutral flux through surrounding surfaces is then calculated from an escape probability formulated from integral transport theory. The concept is to break the outer regions of a diverted tokamak plasma up into a relatively small number of complex geometric regions and to do a balance on the fluxes crossing the surfaces bounding these regions, using surface-to-surface transmission probabilities 
calcula'ed with integral transport theory and region-to-surface escape probabilities based on the repeated application of first-flight transport calculations of escape probabilities. With a few plausible assumptions, the transmission and escape probabilities depend only upon the geometry and the $\mathrm{mfp}$ of the region, so that complex geometries can be represented in precomputed transmission and escape probabilities which can be stored as a function of $\mathrm{mfp}$ and looked up at run time, thereby eliminating the computational penalty of complex geometry. With another plausible assumption, the region-to-surface escape probabilities can be written as simple expressions involving the surface area, volume and $\mathrm{mfp}$ of the region. The most limiting assumption on the method is that of uniform properties (or at least known property variation) within a region, which places a lower limit on the number of regions required.

This paper is organized as follows. Definitions of quantities involved in neutral transport are given in section 2, and a representative geometric model for the outer regions of a diverted tokamak is given in section 3. The fluxes across surfaces are constructed from uncollided fluxes across other surfaces and from collided fluxes emerging from adjacent regions in section 4 . The solution of the resulting flux equations and the use of that solution in a particle balance are indicated in section 5 . The methodology for calculating transmission probabilities, escape probabilities and the plasma albedo are given in sections 6-8, respectively. A discussion of assumptions and applications in section 9 concludes the paper. Explicit expressions for the calculation of transmission probabilities in the model problem geometry are given in appendices.

\section{DEFINITIONS}

$$
\Gamma_{a-b} \quad \equiv \text { neutral flux from region } a \rightarrow \text { region } b
$$




$$
\begin{aligned}
& T_{a-c}^{b} \quad \equiv \text { transmission coefficient expressing the probability that neutral, } \\
& \text { particles incident into region } b \text { from region } a \text { emerge from, } \\
& \text { region } b \text { into region } c \text { without a collision event } \\
& P_{b} \quad \equiv \text { escape probability that a neutral particle "born" in region } b \\
& \text { (external source, charge-exchange event, electron } \\
& \text { recombination) will escape from region } b \text { without being ionized } \\
& \Lambda_{b-c} \equiv \text { probability that a neutral particle "born" in and escaping from } \\
& \text { region } b \text { escapes into adjacent region } c \\
& \alpha_{a b} \quad \equiv \text { albedo of region } b \equiv \text { the probability that a neutral particle } \\
& \text { entering region } b \text { from region a (or its neutral progeny) re- } \\
& \text { emerges from region } b \text { into region } a \\
& R_{w b} \quad \equiv \text { reflection coefficient for wall bounding region } b \equiv \text { probability } \\
& \text { that a neutral (or charged) particle incident upon the wall is } \\
& \text { reflected as a neutral particle. } \\
& f_{p b} \quad \equiv \text { fraction of neutrals striking the "wall" bounding region } b \text { that } \\
& \text { are removed by pumping }
\end{aligned}
$$

\section{MODEL GEOMETRY}

A representative geometry of the plasma, SOL, divertor and plenum regions within the plasma and divertor chambers is illustrated for a axisymmetric tokamak with a single null divertor configuration in Fig. 1.

A simple geometric model which should represent the actual geometry of Fig. 1 reasonably well is illustrated in Fig. 2 . The divertor channel has been divided into a presheath region ( 1 and 2 ) which extends a few $\mathrm{mfp}$ in front of the collector plate, in which intense neutral-charged particle interactions take place, and a channel (3 and 4) extending up to the throat (5). All of these regions can be represented by trapezoids. The divertor plenum (6-8) can be represented rather exactly by regions bounded by 
straight lines. The SOL (9-12) and the plasma chamber (13-16) can be represented approximately by concentric annular segments to allow for treating poloidal asymmetries. The model of Fig. 2 is for the purpose of illustration--the actual geometric model that would be used in any given calculation would depend on the configuration and the geometrical accuracy required.

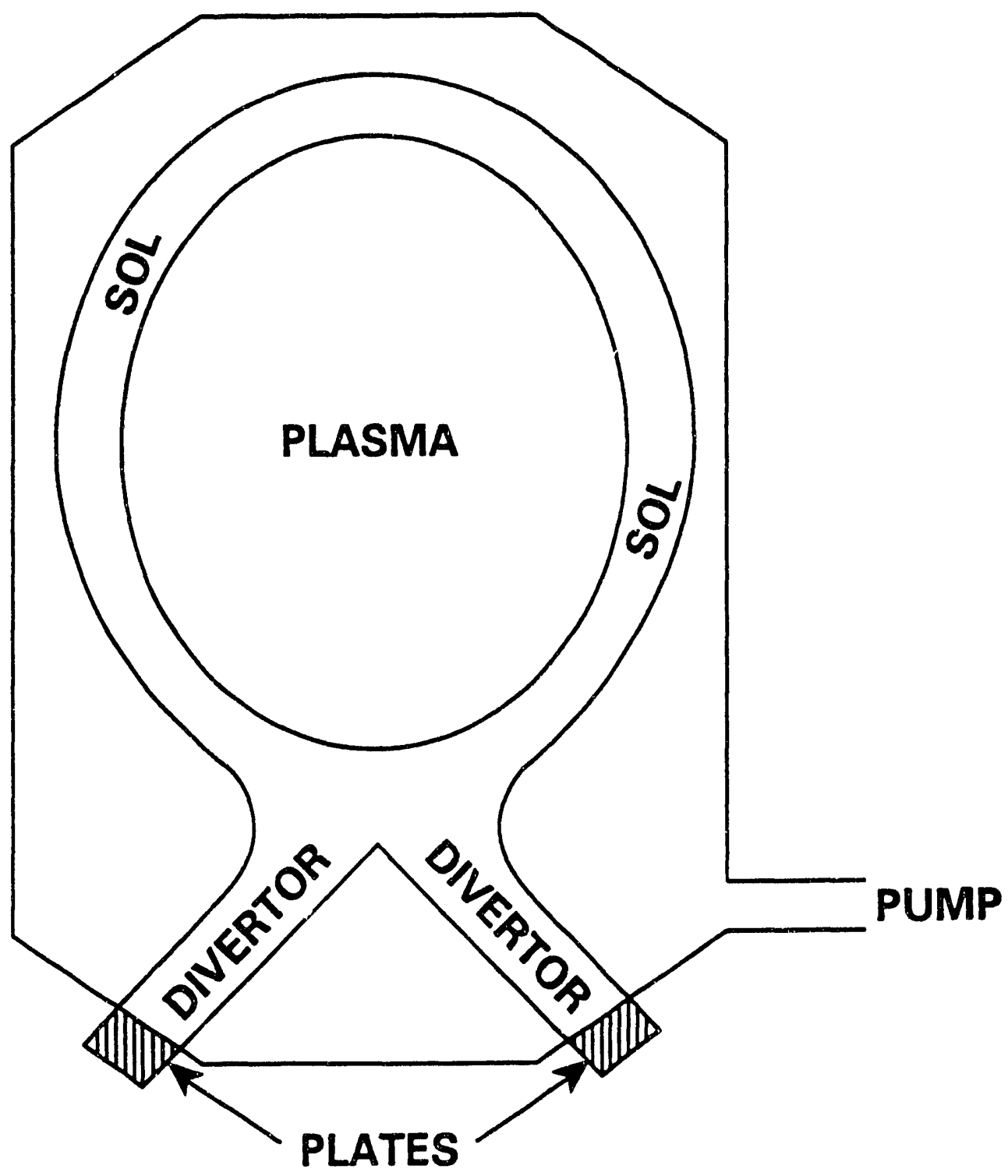

Fig. 1: Single Null Diverter Geometry (Not to scale) 


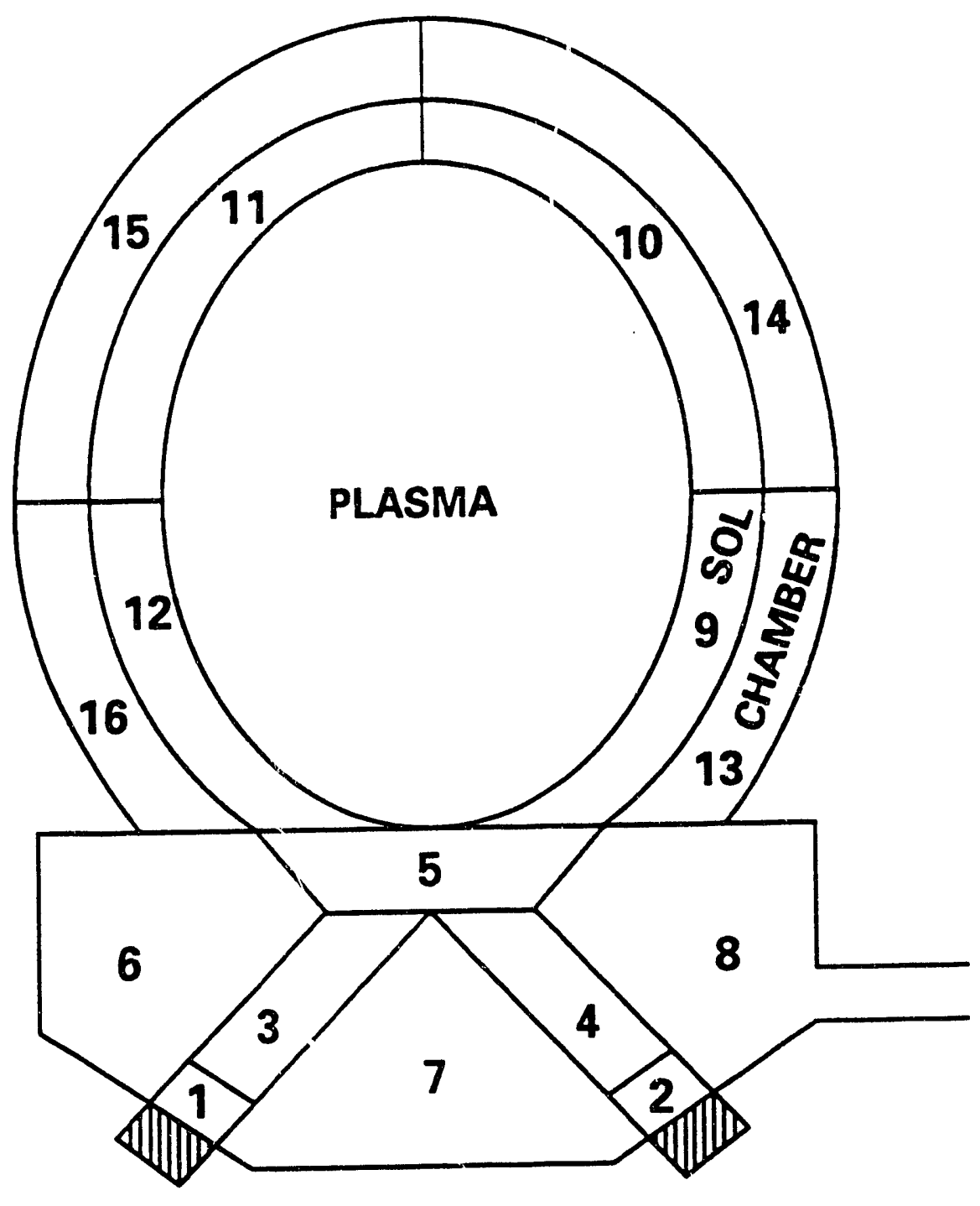

Fig. 2: Geometric Model for Outer

Regions Neutral Calculation 


\section{FLUXES ACROSS SURFACES}

The formalism is developed for a neutral hydrogenic atomic species. Modifications to allow treatment of a neutral impurity species are discussed in a subsequent section.

With respect to Fig. 2, the neutral flux emerging from region $i$ into adjacent region $j$ consists of: 1) the suri of the neutral fluxes that entered region $i$ from all adjacent regions times the probabilities that these fluxes emerge without collision into region $j$

$$
\sum_{k}^{i} \Gamma_{k-i} T_{k-j}^{i}
$$

where $\sum_{k}^{i} \equiv$ the sum over all regions $k$ that are contiguous to region $i$; plus 2 ) the sum of all neutral fluxes that entered region $i$ from all adjacent regions and that had a collision in region $i$

$$
\sum_{k}^{i} \Gamma_{k-i}\left(1-\sum_{l}^{i} T_{k-l}^{i}\right)
$$

times the probability $A_{c x}^{i} \equiv \frac{\langle\sigma \mathrm{v}\rangle_{c x}^{i}}{\langle\sigma \mathrm{v}\rangle_{c x}^{i}+\langle\sigma \mathrm{v}\rangle_{i o n}^{i}}$ that the collision was a charge-exchange event, times the probability $\left(P_{i}\right)$ that the charge-exchange neutral or its progeny eventually escapes from region $i$, times the probability $\left(\Lambda_{i-j}\right)$ that a neutral particle escaping from region $i$ enters region $j$; plus 3 ) the probability that a neutral particle introduced into region $i$ externally $\left(S_{i}\right)$ escapes into region $j\left(P_{i} \Lambda_{i-j}\right)$. 


$$
\Gamma_{i-j}=\sum_{k}^{i} \Gamma_{k-i}\left(T_{k-j}^{i}+A_{c x}^{i} P_{i} \Lambda_{i-j}\left(1-\sum_{l}^{i} T_{k-l}^{i}\right)\right)+S_{e x}^{i} P_{i} \Lambda_{i-j}
$$

The flux from a region $i$ in the plasma edge into the adjacent SOL region $j$ is

$$
\Gamma_{i-j}=\alpha_{j i} \Gamma_{j-i}
$$

Thus, it is not necessary to explicitly treat the plasma inside the separatrix in the neutral calculation; rather it is treated as an albedo boundary c sndition on the inner surface of the SOL.

The neutral flux into region $i$ from a bounding wall region $i w$ is the sum of: 1) the neutral flux from region $i$ to the wall, given by Eq. (1), times the probability $\left(1-f_{p i}\right)$ that this incident flux is not pumpe : times the probability $\left(R_{w i}\right)$ that this incident flux is reflected from the wall; plus 2$)$ the ion flux $\left(\Phi_{\text {plate }}^{i w}\right)$ incident upon the divertor plate--for the presheath regions only--times the reflection coefficient $\left(R_{\text {wi }}\right)$

$$
\begin{aligned}
\Gamma_{i w-i} & =\left(1-f_{p i}\right) R_{w i}\left[\sum_{k}^{i} \Gamma_{k-i}\left(T_{k-i w}^{i}+A_{c x}^{i} P_{i} \Lambda_{i-i w}\left(1-\sum_{l}^{i} T_{k-l}^{i}\right)\right)+S_{e x}^{i} P_{i} \Lambda_{i-i w}\right] \\
& +\Phi_{p l a t e}^{i w} R_{w i} \delta_{i w, p s}
\end{aligned}
$$

where $\delta=1$ for the presheath and $=0$ otherwise.

The transmission probabilities, $T_{k-j}^{i}$, involve mean free paths $\lambda=\frac{\mathrm{v}_{o}}{n\langle\sigma \mathrm{v}\rangle}$, which should be evaluated using the neutral speed $\left(v_{o}^{k}\right)$ that is characteristic of the region $k$ from which the neutral has emerged, and using $n_{i}\langle\sigma v\rangle_{i}$ for the region $i$. 


$$
\begin{aligned}
\langle\sigma v\rangle_{i o n} & =\langle\sigma v\rangle_{i o n}^{i}+\frac{n_{e}}{n_{i}}\langle\sigma v\rangle_{i o n}^{e}+\sum_{j} \frac{n_{z j}}{n_{i}}\langle\sigma v\rangle_{c x}^{j} \\
& =\left[\langle\sigma v\rangle_{i o n}^{i}+\left(1+\frac{\alpha}{Z}\right)\langle\sigma v\rangle_{i o n}^{e}+\frac{\alpha}{Z^{2}} \sum_{j} \chi_{z j}\langle\sigma v\rangle_{c x}^{j}\right]
\end{aligned}
$$

where $\alpha \equiv n_{z} Z^{2} / n_{i}$ and $\chi_{z j} \equiv n_{z j} / n_{z}$, with $n_{z}$ and $n_{z j}$ and being the total and $j-t h$ charge state impurity densities, and $\left\langle\sigma_{v}\right\rangle_{c x}^{j}$ is the charge-exchange rate between the neutral bydrogenic species and the $j-t h$ impurity charge state.

\section{SOLUTION STEPS}

With the assumptions: 1) the plasma properties are uniform (or at least vary in a known way) within a region; 2) the incident neutral flux is isotropic over the inward hemisphere; and 3) the incident-neutral flux is uniformly distributed over the surface, the transmission and escape probabilities depend only on mean-free-path and geometry of the region. Since geometry is fixed the transmission and escape probabilities can be precomputed as a function of $\lambda$ and looked up from a table at run time. Fixed, but non-uniform plasma properties and incident neutral flux distribution, and a prespecified anisotropic angular distribution, could be accommodated within a precomputed table, but it is unlikely that the knowledge of these non-unifo. nities and anisotropies would be good enough to make this worthwhile.

$2 \mathrm{~N}$ equations, where $N$ is the number of interfaces, of the type of Eqs. (1)-(3) obtain. These equations have the general form

$$
\underline{\underline{M}}=\underline{S}_{e x}+\Phi_{\text {plate }}
$$

and the general solution

$$
\underline{\Gamma}=\underline{M}^{-1}\left[\underline{S}_{e x}+\underline{\Phi}_{\text {plate }}\right]
$$


The reflected neutral flux consists of two components: 1) a fraction $\chi$ of neutrals that are reflected with a fraction $g$ of the speed of the incident neutral/ion; and 2) a fraction $(1-\chi)$ that thermalize in the wall, are re-emitied as molecules, then dissociate to produce neutrals with the Franck-Condon speed $\mathrm{v}_{c}^{F C}$

$$
\Gamma_{i w-i}=\chi \Gamma_{i w-i}^{(g)}+\Gamma_{i \mathrm{H}-i}^{(F C)}(1-\chi)
$$

When calculating the transmission probabilities for these reflected neutrals across presheath region $i$, a neutral speed $g \mathrm{v}_{o}^{i}$ should be used to evaluate the $\mathrm{mfp}$ for the first component, and a neutral speed $v_{o}^{F C}$ should be used to evaluate the mfp for the second component.

The total ionization rate in region $i$ consists of the ionization of external source neutrals and of neutrals flowing into region $i$ from adjacent regions, after zero, one, two, three, ... charge-exchange events

$$
\begin{aligned}
I_{i} & =\left[S_{e x}^{i}+\sum_{k}^{i} \Gamma_{k-i}\left(1-\sum_{l}^{i} T_{k-l}^{i}\right)\right] A_{i o n}^{i} \sum_{n=0}^{\infty}\left[A_{c x}^{i}\left(1-F_{i}\right)\right]^{n} \\
& =\frac{\left[S_{e x}^{i}+\sum_{k}^{i} \Gamma_{k-i}\left(1-\sum_{l}^{i} T_{k-l}^{i}\right)\right] A_{i o n}^{i}}{1-A_{c x}^{i}\left(1-P_{i}\right)}
\end{aligned}
$$

where $A_{\text {ion }}^{i} \equiv 1-A_{c x}^{i}$.

The presence of impurities is taken into account by defining an effective ionization rate in terms of the plasma ion concentration, $n_{i}$ 
The neutral particle balance equation in each region is

$$
\dot{N}_{j}=S_{e x}^{i}-\sum_{k}^{j}\left(\Gamma_{j-k}-\Gamma_{k-j}\right)-N^{i o n}\langle\sigma \mathrm{v}\rangle_{\text {ion }}^{j} N_{j}
$$

which has the steady-state solution

$$
N_{j}=\frac{S_{e x}^{j}-\sum_{k}^{j}\left(\Gamma_{j-k}-\Gamma_{k-j}\right)}{N^{i o n}\langle\sigma v\rangle_{i o n}^{j}}
$$

The numerical solution steps are:

1) evaluate the transmission and escape probabilities from precomputed tables, using the plasma densities and temperatures to calculate $\lambda$;

2) solve Eq. (7) for $\underline{\Gamma}$ by inverting $\underline{\underline{M}}$;

3) use $\underline{\Gamma}$ in Eq. (10) to obtain the steady-state neutral content or in Eq. (9) to advance the dynamic solution.

\section{TRANSMISSION PROBABILITY}

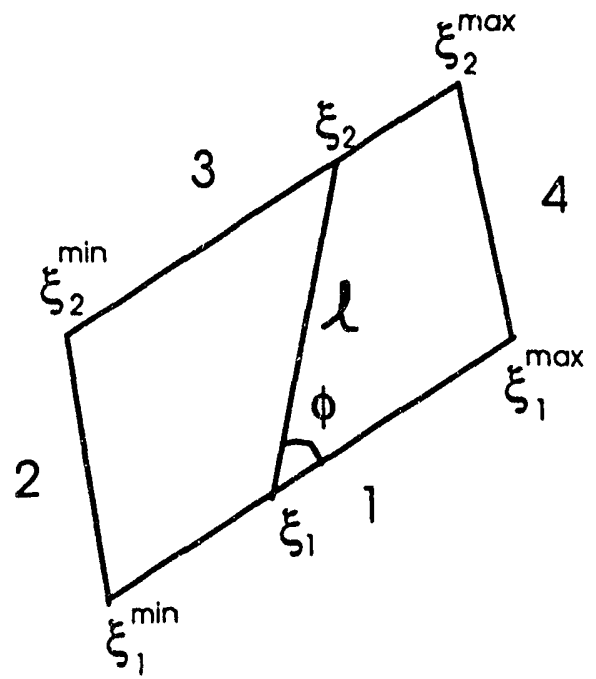

Fig. 3: Surface-to-Surface Transmission 
With reference to Fig. 3, we are interested in calculating the probability that a flux of neutrals incident upon side 1 of the region emerges uncollided through side 3 , for example. We call this probability the transmission probability $T_{1-3}$.

The uncollided neutral flux is given by

$$
\varphi(l)=\varphi(o) e^{-l / \lambda}
$$

where

$$
\lambda \equiv \frac{\mathbf{v}_{o}}{n_{i}\left(\langle\sigma v\rangle_{i o n}+\langle\sigma v\rangle_{c x}\right)}
$$

and $l$ is the path length from the point of entry into the region.

If the distribution of the incident flux along side 1 is $\Gamma_{1}\left(\xi_{1}, \phi\right)$, then the transmission probability is

$$
T_{1-3} \equiv \frac{\int_{\xi_{1}^{\min }}^{\xi_{1}^{\max }} d \xi_{1} \int_{\phi_{\min }\left(\xi_{1}\right)}^{\phi_{\max }\left(\xi_{1}\right)} d \phi e^{-l(\phi) / \lambda} \Gamma_{1}\left(\xi_{1}, \phi\right)}{\int_{\xi_{1}^{\min }}^{\xi_{\max }} d \xi_{1} \int_{0}^{\pi} d \phi \Gamma_{1}\left(\xi_{1}, \phi\right)}
$$

The angles $\phi_{\min }\left(\xi_{1}\right)$ and $\phi_{\max }\left(\xi_{1}\right)$ are the minimum and maximum angles $\phi$ subtended at $\xi_{1}$ by the side 3 . Now $l(\phi)$ represents the path length from point $\xi_{1}$ on side 1 to point $\xi_{2}$ on side 2, as shown in Fig. (3).

If the incident particle distribution is isotropic over the inward hemisphere, then $\Gamma_{1}\left(\xi_{1}, \phi\right) \rightarrow \Gamma_{1}\left(\xi_{1}\right) / \pi$ and Eq. (13) reduces to

$$
T_{1-3}=\equiv \frac{\int_{\xi_{1}^{\min }}^{\xi_{1}^{\max }} d \xi_{1} \Gamma_{1}\left(\xi_{1}\right) \frac{1}{\pi} \int_{\phi_{\min }}^{\phi_{\max }\left(\xi_{1}\right)} d \phi e^{-l(\phi) / \lambda}}{\int_{\xi_{1}^{\min }}^{\xi_{1}^{\max }} d \xi_{1} \Gamma_{1}\left(\xi_{1}\right)}
$$


We further define the local transmission probability that an isotropically distributed neutral incident at point $\xi_{1}$ on side 1 emerges uncollided through side 3

$$
t_{1-3}\left(\xi_{1}\right) \equiv \frac{1}{\pi} \int_{\phi_{\min }\left(\xi_{1}\right)}^{\phi_{\max }\left(\xi_{1}\right)} d \phi e^{-l(\phi) / \lambda}
$$

If the incident flux is uniformly distributed along side 1 , then the transmission probability reduces to

$$
T_{1-3}=\int_{\xi_{1}^{\min }}^{\xi_{1}^{\max }} d \xi_{1} t_{1-3}\left(\xi_{1}\right) /\left(\xi_{1}^{\max }-\xi_{1}^{\min }\right)
$$

For a fixed geometry, this quantity depends only on $\lambda$. Transmission probabilities are calculated in the appendices for the geometry of Fig. 2.

\section{ESCAPE PROBABILITY}

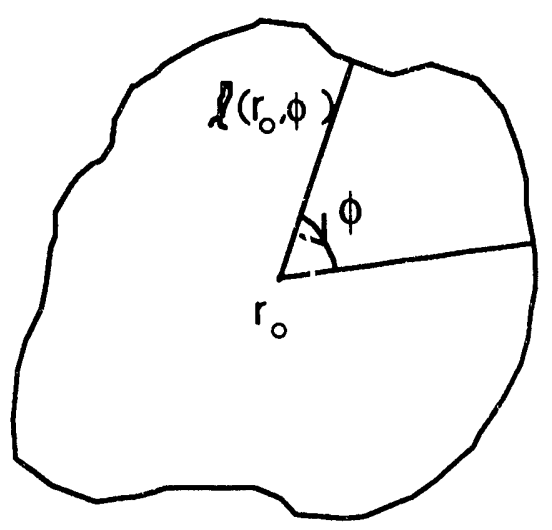

Fig. 4: Escape Probability Geometry

A neutral particle introduced externally or created by charge-exchange, with an isotropic distribution, at point $r_{o}$ has a probability

$$
P_{o}^{\prime}\left(r_{o}\right)=\frac{1}{2 \pi} \int_{o}^{2 \pi} e^{-l\left(r_{o}, \phi\right) / \lambda} d \phi
$$


of escaping from the region uncollided, where now $l\left(r_{o}, \phi\right)$ is the chord length from $r_{o}$ to the surface in the direction denoted by $\phi$.

If $S\left(r_{o}\right)$ is the distribution of particles 'born" in the region, then

$$
P_{o} \equiv \int d r_{o} S\left(r_{o}\right) P_{o}^{\prime}\left(r_{o}\right) / \int d r_{o} S\left(r_{o}\right)
$$

is the average probability that a particle born in the region will escape without having a collision.

If $S\left(r_{o}\right)=$ const., $P_{o}$ may be evaluated analytically or numerically. Results are given in Ref. [2] for a number of simple shapes. When the dimensions of the region are large relative to the $\mathrm{mfp}$, considerations of average chord length and reciprocity [2] lead to the simple approximation

$$
P_{o} \simeq \frac{S \lambda}{4 \mathrm{~V}}
$$

where $\lambda$ is given by Eq. (12), and $S$ and $V$ are the surface area and volume of the region. This result is valid when the region is large compared to a mean-free-path, $\lambda\langle\langle 4 \mathrm{~V} / S$. In the other extreme, $\lambda\rangle\rangle 4 \mathrm{~V} / S$, the escape probability must approach unity. This suggests that the rational approximation

$$
\hat{P}_{o}=\frac{1}{1+P_{o}^{-1}} \text {. }
$$

be used to evaluate the escape probability.

The particle may escape from the region uncollided, with probability $P_{0}$; its progeny may escape after one collision, with probability $\left(1-P_{o}\right) A_{c x} P_{o}$; etc. The total escape probability for a neutral particle or its neutral progeny is

$$
\begin{aligned}
P & =P_{o}+\left(1-P_{o}\right) A_{c x}+\left(1-P_{o}\right)^{2}\left(A_{c x}\right)^{2} P_{o}+\ldots \\
& =P_{o} \sum_{n=0}^{\infty}\left(A_{c x}\left(1-P_{o}\right)\right)^{n}=\frac{P_{o}}{1-A_{c x}\left(1-P_{o}\right)}
\end{aligned}
$$


The distribution of escaping neutral particles among contiguous regions can be estimated from considerations of surface area (length in a 2D model) and the motion of the progenitor ion in the case of a charge-exchange neutral. An ion moves along a field line with its thermal speed; if there is a net ion flow, $\overline{\mathrm{v}}_{\|}$, then

$$
n \overline{\mathrm{v}}_{\|}=\int_{-\infty}^{o} f\left(\mathrm{v}_{\|}\right) \mathrm{v}_{\|} d \mathrm{v}_{\|}+\int_{0}^{\infty} f\left(\mathrm{v}_{\|}\right) \mathrm{v}_{\|} d \mathrm{v}_{\|} \equiv-n^{-} \mathrm{v}_{t h}+n^{+} \mathrm{v}_{\text {th }}
$$

where $n^{+}$and $n^{-}$are the number of ions moving parallel and anti-parallel, respectively, to the field direction. Ion motion perpendicular to the field line has no preferential direction. Thus, the relative probability that a charge-exchange neutral will escape from $i$ into region $j$ depends on the relative length $(L i j)$ of the interface between regions $i$ and $j$ and on the orientation of that interface with respect to the magnetic field

$$
\Lambda_{i-j}=\text { const. } L_{i j}\left(1+\left(\hat{n}_{\|} \cdot \hat{n}_{i j}\right) \bar{\nabla}_{\|} / v_{t h}\right)
$$

where $\hat{n}_{\|}$is the unit vector along the field line and $\hat{n}_{i j}$ is the normal unit vector to $L_{i j}$ pointing from region $i$ to region $j$. The constant is determined from the requirement $\sum_{j}^{i} \Lambda_{i-j}=1$

\section{PLASMA ALBEDO}

Neutrals crossing the separatrix from the SOL into the plasma edge region will either be ionized immediately or will undergo a sequence of charge-exchange events terminating in ionization, with the result that a negligible number of neutrals penetrate beyond the plasma edge region. However, some of the charge-exchange neutrals will re-emerge from the plasma edge back into the SOL. 
Treating the plasma as an infinite half space for neutrals entering from the SOL and making use of the diffusion theory approximation, the plasma albedo is, by analogy to the neutron diffusion theory result [14],

$$
\alpha_{e d g e-S O L} \equiv \frac{\Gamma_{e d g e-S O L}}{\Gamma_{S O L-e d g e}}=\frac{1-\frac{2}{\sqrt{3}}\left(A_{c x}^{-1}-1\right)^{1 / 2}}{1+\frac{2}{\sqrt{3}}\left(A_{c x}^{-1}-1\right)^{1 / 2}},
$$

where $A_{c x}$ is the charge-exch thige probability in the plasma edge region.

\section{MOD!FICATIONS TO TREAT A NEUTRAL IMPURITY SPECIES}

The principal modification of the above formalism that is required in order to treat the transport of neutral impurity atoms is the replacement of the expression for the neutral flux from the wall irito region $i$ given by Eq. (3) with

$$
\begin{aligned}
\Gamma_{i w-i}^{z}= & \sum_{a}\left\{( 1 - f _ { p i } ^ { a } ) Y _ { w i } ^ { a - z } \left[\sum _ { k } ^ { i } \Gamma _ { k - i } ^ { a } \left(T_{k-i w}^{i, a}+\right.\right.\right. \\
& \left.\left.\left.A_{c x}^{i, a} P_{i}^{a} \Lambda_{i-i w}^{a}\left(1-\sum_{l}^{i} T_{k-l}^{i, a}\right)\right)+S_{e x}^{i, a} P_{i}^{a} \Lambda_{i-i w}^{a}\right]\right\}+ \\
& \sum_{b} \Phi_{p l a t e}^{i w, b} Y_{w i}^{b-z} \delta_{i w, p s}
\end{aligned}
$$

The sums over $a$ and $b$ are over all neutral and ionic species, respectively. The quantities $Y_{w i}^{a-z}$ and $Y_{w i}^{b-z}$ are the sputtering yields of impurity neutrals of species z per neutral of species $a$ or ion of species $b$, respectively, striking the wall of region $i$. Equation (25) can be generalized to have a different sputtering yield for each component of the neutral flux to the wall, evaluated for the energy corresponding to 
the region of origin of the neutral flux component. The other quantities have been defined previously without the species-dependent superscripts $a$ and $b$.

\section{DISCUSSION}

The appeal of the proposed model is its ability to accurately calculate neutral transport in a complex $2 \mathrm{D}$ geometry with relatively few equations, because the geometrical complexity enters via transmission and escape probabilities which can be precomputed as a function of $\mathrm{mfp}$ and obtained from tables at run time. The geometrical model of Fig. 2 serves to quantify the number of equations that might be involved in a typical application. There are 2 flux equations for each "internal" surface and 1 flux equation for each "external" surface bounded by either the plasma or the wall, for a total of 64 equations. The coupling among surface fluxes is nearestneighbor, so the matrix M of Eq. (7) is relatively sparse.

While the geometric model of Fig. 2 is composed of regions that are bounded by straight lines and arcs of circles, and the transmission probabilities given in the appendices are for such a geometry, this is not a limitation on the model. Once the geometry is specified, no matter how complex, the transmission and escape probabilities can be precomputed numerically and only a table lookup is needed at run time.

The transmission probabilities are exact, subject to three assumptions: 1) uniform $\mathrm{mfp} ; 2$ ) isotropic angular distribution of the incident flux over the inward hemisphere; and 3) uniform distribution of the incident flux over the surface. All of these assumptions can be removed by generalizing to known variations. The first assumption is not so limiting as it appears to be, since the transmission calculated with an exponentially varying density between two surfaces is the same as would be calculated using the average density. The second assumption should be valid except 
near corners joining regions of very different $\mathrm{mfp}$. The consequences of the third assumption would be ameliorated by subdividing surfaces, if necessary.

The first-flight escape probabilities are exact, subject to three assumptions: 1) uniform $\mathrm{mfp}$; 2) isotropic angular distribution of "secondary" charge-exchange and external source neutrals; and 3) uniform spatial distribution of charge-exchange and external source neutrals. Again, the first assumption is not as limiting as it seems. The second assumption is quite plausible. The third assumption is perhaps questionable for virgin source neutrals and first-collision charge-exchange neutrals in some regions, but is quite plausible for those neutrals escaping after two or more charge-exchange events. Note, however, that the simple expression $P_{o} \simeq \frac{S \lambda}{4 \mathrm{~V}}$ is subject to the further assumption that the region is large compared to a $\mathrm{mfp}$.

The methodology proposed in this paper lends itself to taking into account differences in the speeds of neutrals that originate in different regions and thus using the correct mean free paths in determining transmission and attenuation. This is an intrinsic advantage of an integral transport based method over a differential transport based method, which would require an additional modeling of energy transfer (e.g. a multigroup model).

It is felt that the methodology proposed in this paper can lead to both relatively simple neutral transport models that can be used with simple "2-point" plasma models of the SOL and divertor for scoping and parameter studies and to detailed neutral transport models that can be used with $1 D$ and 2D plasma models of the SOL and divertor for detailed analysis of experiment and divertor design. Such models should be able to obtain greater accuracy for a given computational time than other models presently available for the calculation of neutral transport in the outer regions of a diverted tokamak. Computational studies in support of this conjecture will be published in the future. 


\section{REFERENCES}

1. M. Tendler, D. Heifetz, "Neutral Particle Kinetics in Fusion Devices", Fusion Techn., 11,289 (1987).

2. K. M. Case, F. deHoffman, G. Placzek, "Introduction to the Theory of Neutron Diffusion, Vol I", Los Alamos Scientific Laboratory report (1953).

3. B. J. Braams, in "11th European Conf. on Controlled Fusion and Plasma Physics", Aachen, EPS (Petit-Lancy Switzerland) p. 434 (1984); also in "12th European Conf. on Controlled Fusion and Plasma Physics", Budapest, EPS (Petit-Lancy, Switzerland) p. 480 (1985).

4. E. M. Gelbard, "Spherical Harmonics Methods: $\mathrm{P}_{\mathrm{L}}$ and Double- $\mathrm{P}_{\mathrm{L}}$ Approximations", in Computing Methods in Reactor Physics, ed. H. Greenspan C. N. Kelber, D. Okrent, Gordon and Breach, New York (1963), pp. 271-364.

5. B. G. Carlson, K. D. Lathrop, "Transport Theory: The Method of Discrete Ordinates", ibid., pp. 171-270.

6. A. Hassitt, "Diffusion Theory in Two and Three Dimensions", ibid., pp. 91-170

7. E. Greenspan, "Calculation of the Transport of Neutral Atoms in Highly Ionized Plasmas Using Neutron Transport Methods", Nucl. Fusion, 14, 771 (1974).

8. J. H. Marable, E. M. Oblow, "The Application of Neutron Transport Codes to the Transport of Neutrals in Plasmas", Nucl. Sci. \& Engr., 61, 90 (1976).

9. J. G. Gilligan, S. L. Gralnick, W. G. Price, T. Kammash, "Multigroup Calculations of Low-Energy Neutral Transport in Tokamak Plasmas", Nucl. Fusion, 18, 63 (1978).

10. E. L. Vold, A. K. Prinja, F. Najmabadi, R. W. Conn, "The Neutral Diffusion Approximation in a Consistent Tokamak Edge Plasma-Neutral Computation", J. Nucl. Mat., 176-177, 570 (1990).

11. M. H. Kalos, F. R. Nakache, J. Celnik, "Monte Carlo Methods in Reactor Computations", in Computing Methods in Reactor Physics, ed. H. Greenspan, C. N. Kelber, D. Okrent, Gordon and Breach, New York, (1968), pp. 365-443..

12. D. Heifetz, D. Post, M. Petrovic, J. Weisheit, G. Bateman, "A Monte Carlo Model of Neutral Particle Transport in Diverted Plasmas", J. Comp. Phys., 46, 309 (1982). 
13. E. Cupini, A. DeMatteis, R. Simonini, "NIMBUS-Monte Carlo Simulation of Neutral Particle Transport in Fusion Devices", NET report EVR X11-324/9 (1984).

14. R. V. Meghreblian, D. K. Holmes, Reactor Analysis, McGraw-Hill, New York (1960), pp. 193-195. 


\section{APPENDIX A: TRANSMISSION PROBABILITIES FOR}

\section{STRAIGHTLINE GEOMETRIES}

For a region bounded by straight line segments, two situations can be distinguished: 1) transmission between adjacent, intersecting sides; and 2) transmission between non-adjacent, non-intersecting sides. The transmission probabilities are calculated under the assumption that the incident flux is isotropic over the incident hemisphere and uniform over the incident surface.

\section{Adjacent. Intersecting Sides with Included Angle $\theta_{i j}$}

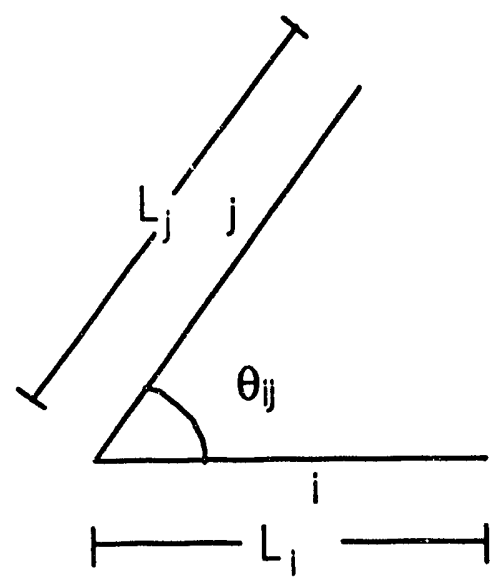

Fig. A.1: Adjacent Side

The local transmission probability for a point $\left(\xi_{i}\right)$ on side $i$ to side $j$, as defined by Eq. (15), is given by

$$
t_{i-j}\left(\xi_{i}\right)=\frac{1}{\pi} \int_{0}^{L_{j}} d \xi_{j} e^{-\xi_{j} \sin \theta_{i j} / \lambda \sin \phi_{1}}
$$

where

$$
\phi_{1}\left(\xi_{i}, \xi_{j}\right)=\cot ^{-1}\left[\frac{\xi_{j}}{\xi_{i} \sin \theta_{i j}}-\cot \theta_{i j}\right]
$$


and $\left(\xi_{i}\right)$ is the distance along side $i$ measured from the intersection.

\section{Non-Adiacent.Non-Intersecting Sides}

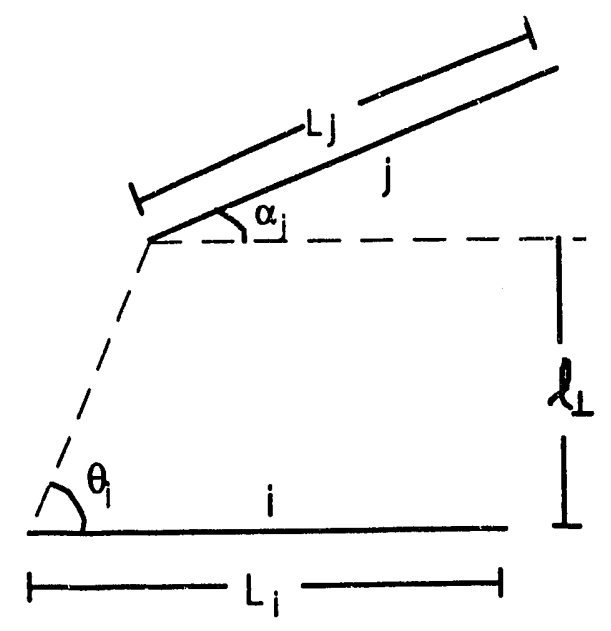

Fig. A.2: Non-Adjacent Sides

The local transmission probability, given by Eq. (15), from a point $\left(\xi_{i}\right)$ on side $i$ to side $j$ is given by

$$
t_{i-j}\left(\xi_{i}\right)=\frac{1}{\pi} \int_{o}^{L_{j}} d \xi_{j} e^{-\left(l_{\perp}+\xi_{j} \sin \alpha_{j}\right) / \lambda \sin \phi_{2}}
$$

where

$$
\phi_{2}\left(\xi_{i}, \xi_{j}\right)=\cot ^{-1}\left[\frac{\xi_{j} \cos \alpha_{j}-\xi_{i}+l_{\perp} \cot \theta_{i}}{l_{\perp}+\xi_{j} \sin \alpha_{j}}\right]
$$

and $\xi_{j}$ is measured along side $j$ from left to right. 
The total transmission probability of Eq. (16) is given by

$$
T_{i-j}=\int_{0}^{L_{i}} d \xi_{i} t_{i-j}\left(\xi_{i}\right) / L_{i}
$$

for both cases. 


\section{APPENDIX B: TRANSMISSION PROBABILITIES FOR}

ANNULAR SEGMENTS

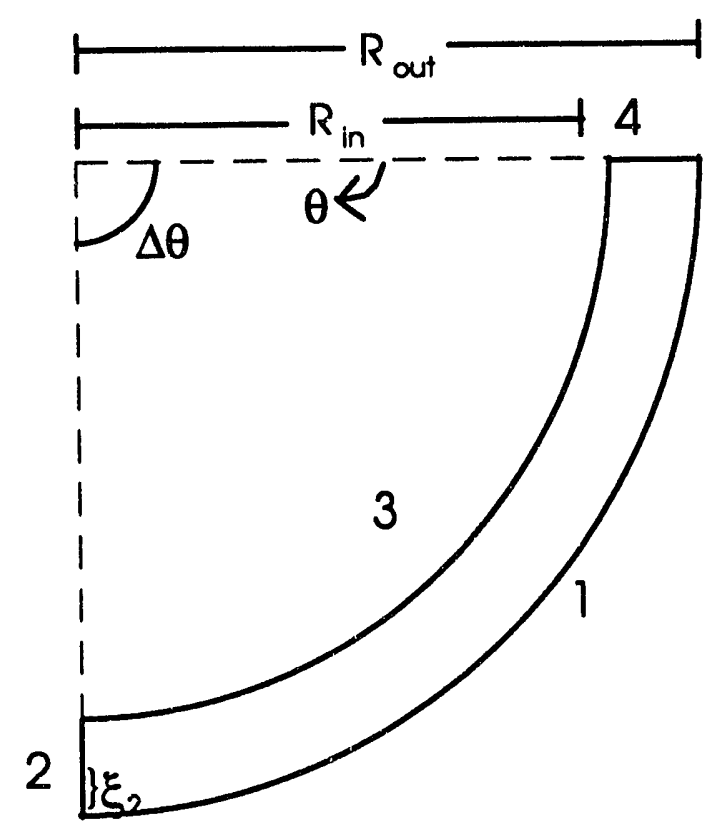

Fig. B.1: Annular Segment Geometry

The geometry of an annular segment can be characterized by the angle $(\Delta \theta)$ subtended by the segment and by the inner $\left(R_{\text {in }}\right)$ and outer $\left(R_{\text {out }}\right)$ radii of the segment. The calculation of transmission probabilities must take into account the facts that some points on each surface cannot be "seen" by a straight line-of-sight from some points on another surface and that there is transmission from an inwardly concave outer surface to itself. The transmission probabilities are calculated under the assumptions that the angular distribution of the incident flux is isotropic over the inward hemisphere and that the spatial distribution of the incident flux is uniform over the surface. Fig. B.1 illustrates the annular segment geometry. 


\section{Transmission from an Outer, Concave-Inward Surface (i.e. side 1)}

The local self-transmission probability, defined by Eq. (12), for a point $\left(R_{\text {out }}, \theta\right)$ on the outer surface 1 to surface 1 is given by

$$
t_{1-1}(\theta)=\frac{1}{\pi} \int_{\theta_{\min }^{11}(\theta)}^{\theta_{\max }^{11}(\theta)} d \theta^{\prime} e^{-2 R_{\text {out }} \sin \left(\frac{\theta-\theta^{\prime}}{2}\right) / \lambda}
$$

where

$$
\begin{aligned}
& \theta_{\min }^{11}(\theta)=\text { larger of } 0 \text { or } \theta+2 \cos ^{-1}\left(\frac{R_{\text {in }}}{R_{\text {out }}}\right) \\
& \theta_{\max }^{11}(\theta)=\text { lesser of } \Delta \theta \text { or } \theta+2 \cos ^{-1}\left(\frac{R_{\text {in }}}{R_{\text {out }}}\right)
\end{aligned}
$$

The local transmission probability from a point $\left(R_{\text {out }}, \theta\right)$ on the outer surface 1 to the radial surface 4 is given by

$$
t_{1-4}(\theta)=\frac{1}{\pi} \int_{\phi_{\min }^{14}}^{\phi_{\max }^{14}(\theta)} d \phi e^{-R_{\text {out }} \sin \theta / \lambda \sin (\phi+\theta)}
$$

where

$$
\begin{aligned}
& \phi_{\min }^{14}(\theta)=\text { larger of }\left(\frac{\pi}{2}-\cos ^{-1} \frac{R_{\text {in }}}{R_{\text {out }}}\right) \text { or } \cot ^{-1}\left[\frac{R_{\text {out }}-R_{\text {in }}}{R_{\text {in }} \sin \theta}+2 \sin ^{2} \frac{\theta}{2}\right] \\
& \phi_{\max }^{14}(\theta)=\text { larger of } \phi_{\min }^{14}(\theta) \text { or }\left(\frac{\pi}{2}-\frac{\theta}{2}\right)
\end{aligned}
$$

The local transmission probability to the other radial surface 2 is obtained from Eqs. (B.3) and (B.4) by replacing $\theta \rightarrow \Delta \theta-\theta$ and $4 \rightarrow 2$.

The local transmission probability from a point $\left(R_{\text {out }}, \theta\right)$ on the outer surface 1 to the inner surface 3 is given by

$$
t_{1-3}(\theta)=\frac{1}{\pi} \int_{\theta_{\min }^{13}(\theta)}^{\theta_{\max }^{13}(\theta)} d \theta^{\prime} e^{-R_{\text {in }} \sin \left(\theta-\theta^{\prime}\right) / \lambda \sin \phi^{13}\left(\theta, \theta^{\prime}\right)-e}
$$


where

$$
\phi^{13}\left(\theta, \theta^{\prime}\right)=\cot ^{-1}\left[\frac{R_{\text {out }}}{R_{\text {in }} \sin \left(\theta-\theta^{\prime}\right)}-\cot \left(\theta-\theta^{\prime}\right)\right]
$$

and

$$
\begin{aligned}
& \theta_{\min }^{13}(\theta)=\text { larger of } 0 \text { or }\left(\theta+\sin ^{-1}\left(\frac{R \text { in }}{R_{\text {out }}}\right)-\frac{\pi}{2}\right) \\
& \theta_{\max }^{13}(\theta)=\text { lesser of } \Delta \theta \text { or }\left(\theta-\sin ^{-1}\left(\frac{R_{\text {in }}}{R_{\text {out }}}\right)+\frac{\pi}{2}\right)
\end{aligned}
$$

The total transmission probabilities, given by Eq. (16), for transmission from the outer surface to surface $j$ are evaluated from

$$
T_{1-j}=\int_{0}^{\Delta \theta} d \theta t_{1-j}(\theta) / \Delta \theta
$$

\section{Transmission from an Inner, Convex-Inward Surface (i.e. side 3)}

The local transmission probability from a point $\left(R_{i n}, \theta\right)$ on the inner surface 3 to the radial surface 4 is given by

$$
t_{3-4}(\theta)=\frac{1}{\pi} \int_{\phi_{\min }^{34}}^{\phi_{\max }^{34}(\theta)} d \phi e^{-R_{i n} \sin \theta / \lambda \sin (\phi+\theta)}
$$

where 


$$
\begin{aligned}
& \phi_{\min }^{34}(\theta)=\frac{\pi}{2} \\
& \phi_{\max }^{34}(\theta)=\text { larger of } \frac{\pi}{2} \text { or } \cot ^{-1}\left[\frac{R_{\text {in }}}{R_{\text {out }} \sin \theta}-\cot \theta\right]
\end{aligned}
$$

The local transmission probability from a point on the inner surface to the other radial surface 2 is given by Eqs. (B.9)-(B.10) with the replacement $\theta \rightarrow \Delta \theta-\theta$ and $4 \rightarrow 2$.

The local transmission probability from a point $\left(R_{i n}, \theta\right)$ on the inner surface 3 to the outer surface 1 is given by

$$
t_{3-1}(\theta)=\frac{1}{\pi} \int_{\theta_{\min }}^{\theta_{\max }^{31}(\theta)} d \theta e^{-R_{\text {out }} \sin \left(\theta-\theta^{\prime}\right) / \lambda \cos \phi^{31}\left(\theta, \theta^{\prime}\right)}
$$

where

$$
\phi^{31}\left(\theta, \theta^{\prime}\right)=\tan ^{-1}\left[\frac{R_{\text {in }}}{R_{\text {out }} \cos \left(\theta, \theta^{\prime}\right)}-\tan \left(\theta, \theta^{\prime}\right)\right]
$$

and

$$
\begin{aligned}
& \theta_{\min }^{31}(\theta)=\text { larger of o or }\left(\theta-\frac{\pi}{2}+\sin ^{-1} \frac{R_{\text {in }}}{R_{\text {out }}}\right) \\
& \theta_{\max }^{31}(\theta)=\text { lesser of } \Delta \text { or }\left(\theta+\frac{\pi}{2}-\sin ^{-1} \frac{R_{\text {in }}}{R_{\text {out }}}\right)
\end{aligned}
$$


The total transmission probability for transmission from the inner surface 3 to a surface $j$ is given by

$$
T_{3-j}=\int_{0}^{\Delta \theta} d \theta t_{3-j}(\theta) / \Delta \theta
$$

\section{Transmission from a Radial Surface (e.g. side 2)}

The local transmission probability for a point $\left(R_{\text {out }}-\xi, \Delta \theta\right)$ on the radial surface 2 to the inner surface 3 is given by

$$
t_{2-3}\left(\xi_{2}\right)=\frac{1}{\pi} \int_{\theta_{\min }^{23}\left(\xi_{2}\right)}^{\Delta \theta} d \theta e^{-\left(R_{\text {out }}-\xi_{2}\right) / \lambda_{23}\left(\sin \phi^{23} \cot (\Delta \theta-\theta)+\cos \phi\right)}
$$

where

$$
\phi^{23}\left(\xi_{2}, \theta\right)=\cot ^{-1}\left[\frac{R_{o u t}-\xi_{2}}{R_{\text {in }} \sin (\Delta \theta-\theta)}-\cot (\Delta \theta-\theta)\right]
$$

and

$$
\theta_{\min }^{23}\left(\xi_{2}\right)=\text { larger of } 0 \text { or } \Delta \theta-\cos ^{-1}\left(\frac{R_{\text {in }}}{R_{\text {out }}-\xi_{2}}\right)
$$

The local transmission probability for a point $\left(R_{\text {out }}-\xi_{2}, \Delta \theta\right)$ on the radial surface 2 to the outer surface 1 is given by

$$
t_{2-1}\left(\xi_{2}\right)=\frac{1}{\pi} \int_{\theta_{\min }^{21}\left(\xi_{2}\right)}^{\Delta \theta} d \theta e^{-R_{2} \sin (\Delta \theta-\theta) / \lambda \sin \phi^{21}}
$$

where 


$$
\phi^{21}\left(\xi_{2}, \theta\right)=\cot ^{-1}\left[\frac{R_{\text {out }}-\xi_{2}}{R_{\text {out }} \sin (\Delta \theta-\theta)}-\cot (\Delta \theta-\theta)\right]
$$

and

$$
\theta_{\min }^{21}\left(\xi_{2}\right)=\text { larger of } 0 \text { or } \Delta \theta-2 \cos ^{-1}\left(\frac{R_{\text {in }}}{R_{\text {out }}-\xi_{2}}\right)
$$

The local transmission probability from a point $\left(R_{\text {out }}-\xi_{2}, \Delta \theta\right)$ on the radial surface 2 to the opposite radial surface 4 is given by

$$
t_{2-4}\left(\xi_{2}\right)=\frac{1}{\pi} \int_{0}^{\xi_{4}^{\max }\left(\xi_{2}\right)} d \xi_{4} e^{-\left(R_{\text {out }}-\xi_{4}\right) \sin \Delta \theta / \lambda \sin \phi^{24}}
$$

where

$$
\phi^{24}\left(\xi_{2}, \xi_{4}\right)=\cot ^{-1}\left[\frac{R_{\text {out }}-\xi_{2}}{\left(R_{\text {out }}-\xi_{4}\right) \sin \Delta \theta}-\cot \Delta \theta\right]
$$

and

$$
\xi_{4}^{\max }\left(\xi_{2}\right)=\text { lesser of }\left(R_{\text {out }}-R_{\text {in }}\right) \text { or }\left(\text { larger of } 0 \text { or }\left(R_{\text {out }}-\frac{R_{\text {in }}}{\sin \left(\phi_{\text {tan }}^{24}+\Delta \theta\right)}\right)\right)
$$

where

$$
\phi_{\tan }^{24}\left(\xi_{2}\right)=\cot ^{-1}\left[-\frac{\sqrt{\left(R_{\text {out }}-\xi_{2}\right)^{2}-R_{\text {in }}^{2}}}{R_{\text {in }}}\right]
$$


The local transmission probability for a point $\left(R_{\text {out }}-\xi_{4}, \theta=0\right)$ on the radial surface 4 to the opposite radial surface 2 is given by Eqs. (B.21) - (B.24) with the replacement $\Delta \theta-\theta \rightarrow \theta$ and the interchange of the 2 and 4 subscripts on $\xi$.

The various total transmission coefficients are given by

$$
T_{2-j}=\int_{0}^{R_{\text {out }}-R_{\text {in }}} d \xi_{2 t_{2-j}}\left(\xi_{2}\right) /\left(R_{\text {out }}-R_{\text {in }}\right)
$$



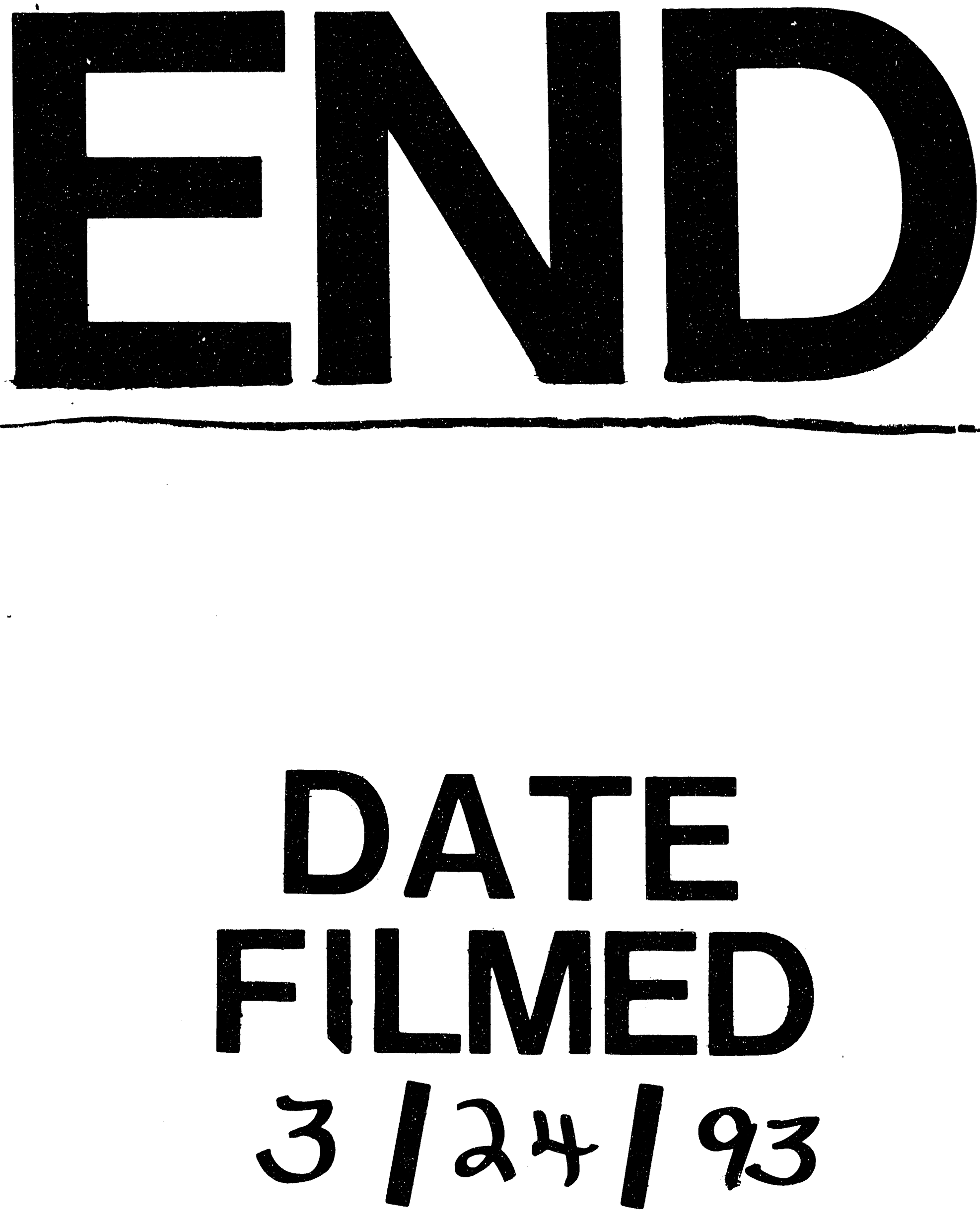
